\title{
Oncopharmacists: The Game Changers in Cancer Therapy
}

\author{
Nandakumar UP, Juno J Joel* \\ Department of Pharmacy Practice, NGSM Institute of Pharmaceutical Sciences, Paneer, NITTE (Deemed to be University), Deralakatte, Mangaluru, Karnataka, INDIA.
}

\begin{abstract}
Cancer is one of the most common cause of morbidity and mortality. Following the diagnosis of the disease, a patient has to undergo complex treatment regimen, which may result in extensive adverse effects. The physical and psychosocial distress experienced by the patients also affects their quality of life. Oncopharmacist can play a crucial role in improving pharmaceutical care for patients undergoing cancer therapy. The day-to-day care provided by the pharmacist will have a positive impact on their quality of life. As they are regarded as the specialists in the field, direct and indirect services provided by them extending from medication administration to drug safety monitoring are valued more.
\end{abstract}

Key words: Oncopharmacists, Cost Minimization, Patient Safety, Cancer,
Clinical Pharmacists.

Correspondence

Dr. Juno J Joel

Assistant Professor, Department of Pharmacy Practice, NGSM Institute of Pharmaceutical Sciences, Paneer, NITTE (Deemed to be University), Deralakatte, Mangaluru-575018, Karnataka, INDIA.

Phone: +919480470727

Email: junojoel@nitte.edu.in

DOI: 10.5530/jyp.2020.12.4

\section{INTRODUCTION}

Cancer is a disease in which abnormal cells divide uncontrollably and may invade the adjacent tissues. Globally it is one of the most common cause of morbidity and mortality and India is of no exception. According to the latest reports of National Institute of Cancer Prevention and Research, it is estimated that around 22.5 lakhs of cancer patients are living in India and approximately 11.5 lakhs of new cases are registered every year. ${ }^{1}$ Early diagnosis of cancer is still a major challenge faced by medical practitioners all over the world. Even after diagnosis, patient has to undergo a complex treatment regimen which comprises of surgical procedures, usage of cytotoxic agents, radiotherapy and other adjuvant therapies. The fundamental principle involved in treatment of cancer is to destruct or arrest the uncontrolled growth of cancerous cells. During the process of destruction of actively dividing cancerous cells, the normal cells of body will get adversely affected, resulting in series of extensive side effects. In addition to the side effects and potential adverse drug reactions, majority of the patients also experience physical, psychological and social distress. The fear of reoccurence among patients also contribute to reduced quality of life. ${ }^{2,3}$

Due to the long duration of therapy, its complex nature and the associated $\mathrm{ADRs}$, leads to the medication non-adherence among the patients. Whereas the patient medication adherence is the primary factor which determines the success of oncotherapy. ${ }^{4,5}$ Oncopharmacist, being specialised in the field of cancer can remain as a sole support with the patient throughout the therapy. The extensive roles handled by oncopharmacist can be discussed

\section{ROLES OF ONCOPHARMACISTS}

\section{Role as a safety officer}

Safety is the foremost concern involved in the treatment of cancer patients, Patients undergoing treatment with anti cancer drugs may encounter various drug realted problems including adverse drug reactions, drug interactions and other techical issues. ${ }^{6}$ Hence oncopharmacists as safety officer always have an essential role to play. Compromise in safety will lead to serious health consequences like prolonged hospitalisation, therapeutic failure, increased health care cost and in extreme conditions, it can even result in death of the patient. ${ }^{7}$ Oncopharmacist as a drug expert have immense knowledge with complete pharmacological and pharmacotherapeutical aspects, which offers him a better oppurtunity to closely design, monitor, assess and modify the therapy if needed. This will lead to prevention, early detection and management of drug related problems encountered. ${ }^{8}$ On other hand, proper documentation and routine/timely reporting of these problems can make other healthcare professionals be aware, which will prevent the reoccurence of such similar events.

\section{Role in reducing polypharmacy}

Combination of drugs are the core component of anti-cancer regimen. Co-prescription of adjuvant and other symptomatic drugs makes the therapy even more complex. Thus polypharmacy directly or indirectly contributes to increased risk of drug related problems. The wise selection of the medications and cutting down the less important medications ease the complexity of regimen and make it more patient friendly. ${ }^{9}$ This might can also decrease the incidence of ADRs which adds up the safety to the regimen.

\section{Role in preparation and administration of medication}

Service by an oncopharmacist can also be extended to the direct patient care, as they receive the anti-cancer drugs. This might can involve several steps including admixing of medication, proper dilution, infusing the drugs after determining the rate of infusion, continuous monitoring of the patient during the course of drug administration and detecting the potential harm to the patients due to therapy. ${ }^{10}$ Meanwhile oncopharmacist critically analyze the patient tolerability towards the drug regimen with proper laboratory references. They are also responsible for verifying and auditing the prescription issued by oncologists and communicate the same with the healthcare team,who are involved in patient care. ${ }^{11}$ 
The above duties by the oncopharmacist helps in achieving a substantial reduction in medication errors.

\section{Role as a policy maker}

The guidelines are the supporting tools for healthcare professionals in delivering quality services. Compliance towards the guidelines not only enhances the effectiveness of therapy, but also reduces the intra and inter personal conflicts. Guidelines are not rigid statements but these are assisting tools in process of healthcare service. ${ }^{12}$ Since they are dynamic, updating the guidelines in any institution is a mandatory requirement. It can be framed by an individual hospital or can be adopted from the existing guidelines of various national and international authorities. Cancer being a global healthcare concern, several guidelines has been proposed. Opinions and suggestions of oncopharmacists has a imperative role in framing such guidelines. ${ }^{13}$ At an institutional level, an oncopharmacist can extend their support to oncologists in determining the ratio in which drugs are to be combined for administration, formulating the storage guidelines, inventory control, preparing and periodic updation of the onco-formulary.

\section{Role in education and counselling patients for medication adherence}

Medication non-adherence remains as a substantial barrier for the success of any treatment regimen. Even though mostly unintentional, many patients tend to neglect taking their medications until they suffer from a negative effect. Since lack of adherence is prevalent even among young cancer patients, it is always important to adopt measures to overcome the issue. ${ }^{4}$ The role of oncopharmacists in enhancing the chemotherapy regimen based knowledge and the importance of medication adherence among patients is widely discussed. ${ }^{5}$ Written information always draw patient attention, leading to improved adherence to medications. Hence, designing and preparation of educationg materials like Patient Information Leaflets (PILs), Pamplets, posters, newsletters etc for cancer is under the scope of oncopharmacist. ${ }^{14}$ Expanded scheme including screening program of cancer, setting up cancer camps and other public awareness campaigns can increase the knowledge level of community which contributes to prevention and early detection of cancer.

\section{Role in research and training}

Cancer is one of the most challenging diseases till date. Still no complete cure is still not available for advanced cancer. ${ }^{15}$ On other hand the DRPs related to the cancer treatment further jeopardize the course of therapy. ${ }^{16}$ Continuous research is an inevitable part to be considered for those diseases with complex therapies. Training and education programs to various members of the cancer care team by demonstration and continuos medical education session serve as better understanding tools. Innovative concept in treatment process and finding out a new safe and effective as well as better tolerable drug molecule is the need of the hour. Collaboration of oncopharmacists with various cancer research organizations at regional, national and international level may give a better breakthrough.

\section{Role as a team member}

Being an expensive therapy it is not always affordable by community people. Cost reduction makes it more affordable and accessible. ${ }^{17} \mathrm{On}$ copharmacists can extend their hands of support to the medical team to deal with the pharmacoeconomical aspects of the disease. In collaboration with doctors and other health care professionals they also can facilitate the easy flow of information and make all of them informed about the additional precautions to be taken with certain category of patients. This facilitates for the substancial reduction in manual errors.

\section{Role as a consultant}

Among the patients suffereing from cancer with other chronic diseases, the number of drugs prescribed usually remains high. ${ }^{18}$ Thus it is obvious that such patients may remain highlty susceptible to drug related issues. Hence the oncopharmacist should carefully evaluate the patient's clinical condition and helps in wise selection of drugs. An oncopharmacist is the right person to select the best possible alternate drug in these special population. Proper collection of medical and medication history before executing the treatment plan is mandatory in case of all patients. Better knowledge on the risk factors, precipitating and predisposing factors aids in precise delivery of therapy.

\section{Role as an oncologist's companion}

Oncology is one among those departments with high patient load in a hospital. Since the ongologists are imposed with heavy work load, it may contribute to the poor and ineffective decision making. Also, the time spent with individual patients will be insufficient to make them understand about their disease or therapy. Oncopharmacists can effectively communicate to oncologist with S-BAR communication, which reduces the possibility of translational errors. Critical interpretation of laboratory data, assessment of patient, clinical and laboratory co-relation and appropriate suggestions on the treatment plan based on laboratory examination by the team pharmacist ultimately contributes to the reduction of physicians workload. Reduction in workload helps in strengthening the rapport between the oncologists and patients. ${ }^{19}$

\section{Role in palliative care}

Pharmacists have a lot to offer for patients with advanced disease undergoing palliative therapy. With regular visits to the patients and care takers, they can address on their fears and medication concerns as well as can provide a moral support. ${ }^{20}$ They can also educate other staffs on pharmacotherapeutic principles of palliative care and can update them with new clinical practice guidelines. They may also evaluate symptomatic complaints of the patient and may recommend appropriate drug therapy. ${ }^{21}$

\section{Role in Medical Reconcilation}

Oncopharmacists performs medical reconcilationas well as they educate and instruct other staffs on good and effective medical reconcilation practices. Role of a pharmacist on MR starts from time of hospital admisssion of a patient, proceeds during the course of therapy and extends upto discharge or transfer to another hospital. ${ }^{22}$

\section{RECOMMENDATIONS}

Services provided by oncopharmacists are inevitable with respect to the better care for patient population as well as a supporting parallel force for the oncologist and other professionals. This review article highlights on the importance of availability of oncopharmacy services. In any cancer speciality hospitals, ocopharmacists shall be enabled to handle the pharmaceuticals related to cancer therapy.Also, there should always be a provision to consider their views and opinionsin developing policies particular to a hospital.

\section{CONCLUSION}

Wide variety of roles ranging from provision of basic education to the level of ensuring patient safety makes oncopharmacists a substantial factor in cancer therapy. As supported by numerous scientific literatures, one can easily quote the importance of oncopharmacist in cancer treatment centres. Almost all scientific research works studying the impact 
of oncopharmacists have concluded on the positive outcomeof their services among cancer patients.

\section{CONFLICT OF INTEREST}

The authors declare no conflict of interest.

\section{ABBREVIATIONS}

ADRs: Adverse Drug Reactions; PILs: Patient Information Leaflets; DRPs: Drug Related Problems; S-BAR: Situation, Background, Assessment and Recommendation; MR: Medical Reconciliation.

\section{REFERENCES}

1. Cancer Statistics. India: National Institute of Cancer Prevention and Research. 2018 [updated 2018 Nov 9; cited 2018 Nov 4]. Available from: http://cancerindia. org.in/cancer-statistics/

2. Chan SY, Dang CC, Amiruddin M, Lai SS, Low CF. An Emerging Role of Pharmacist in Pre-chemotherapy Counseling among Breast Cancer Patients. Indian J Pharm Sci. 2017;79(2):294-302.

3. Schlosser TCM, Ceolim MF. Quality of life of cancer patients during the chemotherapy period. Florianopolis. 2012;21(3):600-7.

4. Chan A, Yap KY. Assessment of the Relationship between Adherence with Antiemetic Drug Therapy and Control of Nausea and Vomiting in Breast Cancer Patients Receiving Anthracycline-Based Chemotherapy. J Manag Care Pharm. 2012;18(5):385-94

5. Perissinotti AJ. Pharmaceutical care for patients receiving cancer treatment. Ann Oncol. 2014;25(5):20.

6. Fornasier G, Taborelli M, Francescon S, Polesel J, Aliberti M, DePaoli P, et al. Targeted therapies and adverse drug reactions in oncology: The role of clinical pharmacist in pharmacovigilance. Int J Clin Pharm. 2018;40(4):795-802.

7. Ma JD, Tran V, Chan C, Mitchell WM, Atayee RS. Retrospective analysis of pharmacist interventions in an ambulatory palliative care practice. $\mathrm{J}$ Oncol Pharm Pract. 2016;22(6):757-65

8. Geissler K. A shared fight against cancer: The complementary roles of oncology physicians and oncology pharmacists. Expert Rev Anticancer Ther. 2013;13(1):13-5

9. Uchida M, Suzuki S, Sugawara H, Suga Y, Kokubun H, Uesawa Y, et al. A nationwide survey of hospital pharmacist interventions to improve polypharmacy for patients with cancer in palliative care in Japan. J Pharm Health Care Sci. 2019;5:14

10. Michaud LB. Managing cancer treatment induced bone loss and osteoporosis in a patient with breast and prostate cancer. Am J Health-Syst Pharm. 2010;67(3):S20-30.

11. Holle LM, Harris CS, Chan A, Fahrenbruch RJ, Labdi BA, Mohs JE, et al. Pharmacist's roles in oncology pharmacy services: Results of a global survey. $J$ Oncol Pharm Prac. 2016;0(0):1-10.

12. Colombo LRP, Ahuir PM, Lima TM. The effects of pharmacist's intervention on adult outpatients with cancer: A systemic review. J Clin Pharm Ther. 2017;42(4):414-24

13. Martini N, Basdew K, kammona A, et al. Pharmacists' views on and experiences with bowel cancer screening kits in Auckland, New Zealand. Int J Pharm Prac. 2014;22(4):257-64

14. Crespo A, Tyszka M. Evaluating the patient-perceived impact of clinical pharmacy services and proactive follow-up care in an ambulatory chemotherapy unit. J Oncol Pharm Prac. 2017;23(4):1-6.

15. Barbour SY. Caring for the treatment-experienced breast cancer patient: The pharmacist's role. Am J Health-Syst Pharm. 2008;65(3):S16-22.

16. Edwards SJ, Abbott R, Edwards J. Outcomes Assessment of a PharmacistDirected Seamless Care Program in an Ambulatory Oncology Clinic. J Pharm Pract. 2014;27(1):46-52.

17. DiScala SL, Onofrio S, Miller M, Nazario M, Silverman M. Integration of a Clinical Pharmacist into an Interdisciplinary Palliative Care Outpatient Clinic. Am J Hosp Palliat Care. 2017;34(9):814-9.

18. Rey JB, Launay-Vacher $V$, Tournigand C. Regorafenib as a single-agent in the treatment of patients with gastrointestinal tumors: An overview for pharmacists. Target Oncol. 2015;10(2):199-213.

19. Scarabelin A, Santana DA, Aguiar PM, Storpirtis S. Pharmacist-patient communication in prostate cancer as a strategy to humanize health care: A qualitative study. J Patient Exp. 2019;6(2):150-6.

20. Atayee RS. Patterns of palliative care pharmacist interventions and outcomes as part of inpatient palliative care consult services. J Pall Med. 2018;21(12):1761-7.

21. Crul M, Oosterhof P. The oncology pharmacist as part of the palliative treatment team. Int J Pharm Prac. 2019;28(1):92-6.

22. Walker KA, Scarpaci L, McPherson ML. Fifty Reasons to love your palliative care Pharmacist. Am J Hosp Palliat Med. 2010;27(8):511-3.

Article History: Submission Date : 01-02-2020 ; Revised Date : 08-02-2020 ; Acceptance Date : 20-02-2020

Cite this article: Nandakumar UP, Joel JJ. Oncopharmacists: The Game Changers in Cancer Therapy. J Young Pharm. 2020;12(1):15-7. 